\title{
¿UTILITARISMO, EMOTIVISMO, DEONTOLOGISMO O ÉTICA DE LA VIRTUD? ESTUDIO DE TRES DILEMAS MORALES APLICADO A ESTUDIANTES BACHILLERES Y UNIVERSITARIOS
}

\author{
UTILITARIANISM, EMOTIVISM, \\ DEONTOLOGY, OR VIRTUE ETHICS? \\ STUDY OF THREE MORAL DILEMMAS APPLIED \\ TO HIGH SCHOOL AND COLLEGE STUDENTS
}

\author{
Fabio Morandín-Ahuerma ${ }^{1}$ \\ https: / / orcid.org/0000-0001-6082-2207 \\ Jaime Salazar-Morales ${ }^{2}$ \\ https: / / orcid.org/ 0000-0003-4393-210X
}

Recibido: mayo 11, 2020 - Aceptado: mayo 28, 2020.

\section{RESUMEN}

En el presente estudio participaron 270 estudiantes en dos muestras; la primera con alumnos de bachillerato, la segunda con alumnos universitarios, ambos del estado de Puebla, México. La investigación fue básicamente cuantitativa, sin embargo, el instrumento utilizado permitió obtener valiosa información cualitativa. El objetivo fue contrastar los resultados históricos de test con dilemas morales clásicos. Los participantes se enfrentaron al Dilema del tranvía, al Dilema de la pasarela y al Dilema de Sofía. Los resultados demostraron que es válida la categorización en cuatro posturas morales básicas: utilitarismo, emotivismo, deontología y ética de la virtud. Y si bien se obtuvieron, en una primera instancia, resultados cuantitativos catalogados como mayormente utilitarios, lo cierto es que las creencias morales exhibidas en el estudio superan el dualismo utilitarismo-emotivismo de estudios previos consultados.

Palabras clave: dilemas morales, posiciones éticas, utilitarismo, deontología, virtudes, estudiantes, México.

1 Posdoctorado en el Centro de Investigaciones Filosóficas (CIF), Programa de Neuroética, Buenos Aires, Argentina. Doctor (cum laude) en Filosofía, Instituto de Filosofía de la Universidad Veracruzana, México. Actualmente es Profesor investigador de tiempo completo de la Benemérita Universidad Autónoma de Puebla, Complejo Regional Nororiental. Candidato a Investigador Nacional en el SNI. fabio.morandin@correo.buap.mx

2 Maestro en Administración y licenciado en Derecho, Benemérita Universidad Autónoma de Puebla, México. Especialista en Filosofía del Derecho y Derecho de las Empresas. Actualmente es Profesor investigador de tiempo completo de la Benemérita Universidad Autónoma de Puebla, Complejo Regional Nororiental, Licenciatura en Derecho, Sede Libres, Puebla. México. jaime.salazarmo@correo.buap.mx 


\section{ABSTRACT}

In the present study, 270 students participated in two samples, the first with high school students, the second with university students, both from the state of Puebla. The research was quantitative, however, the instrument used allowed obtaining valuable qualitative information. The objective was to contrast the historical test results with classic moral dilemmas. Participants faced the Trolley Dilemma, Footbridge Dilemma and Sophia's Dilemma. The results showed that the categorization in four basic moral positions is valid: utilitarianism, emotivism, deontology and virtue ethics. And although quantitative results cataloged as mostly utilitarian were obtained in the first instance, the truth is that the moral beliefs exhibited in the study surpass the utilitarianism-emotivism dualism of previous studies consulted.

Key words: Moral dilemmas, utilitarianism, deontological ethics, virtue ethics, high school and undergraduate students, México.

\section{INTRODUCCIÓN}

La filósofa británica Phillippa Foot publicó, en 1967, un artículo titulado The problem of abortion and the doctrine of double effect [ «El problema del aborto y la doctrina del doble efecto»] donde esbozó una serie de escenarios dilemáticos, entre ellos una primera versión del Dilema del tranvía y analizó algunas implicaciones que las decisiones morales causan en otras personas, consecuencias directas e indirectas de la acción moral y la diferencia, por ejemplo, entre matar y dejar morir. Foot también planteó varios aspectos entre la acción voluntaria e involuntaria; la diferencia entre actuar o permitir, y señaló la obligación moral de proporcionar ayuda a quien la necesita o, al menos, no causar daño a los demás. Su trabajo, indicó, tenía como propósito servir de respuesta a un texto de Herbert Hart, titulado Intention and Punishment [ Intención y castigo»] (Hart, 1967), sobre el problema de la culpabilidad o la inocencia y resolver, de paso, el dilema del determinismo frente al libre albedrío.

Años antes, Elizabeth Anscombe, muy cercana a Foot en la Universidad de Oxford, había publicado otro influyente artículo, Modern Moral Philosophy [«Filosofía moral moderna»] (Anscombe, 1958), en que rescató la importancia de la ética aristotélico-tomista y analizó cómo la acción debe evaluarse desde el punto de vista de su intencionalidad, la voluntad del agente al realizarla, y si se sigue o no un bien moral. Defendió la importancia del carácter de la persona, esto es, su calidad humana como criterio para evaluar lo que se debería hacer (Edmonds, 2013). Esta postura filosófica se denomina ética de la virtud.

Tanto el trabajo de Anscombe, como el de Foot, respondían de alguna manera al ambiente escéptico que prevalecía entre filósofos académicos, como el Circulo de Viena, quienes consideraban, primero, que las proposiciones morales carecían de sentido (Wittgenstein, 1912/2002) y que, en todo caso, deberían evaluarse las acciones por sus efectos y consecuencias (Mill, 1863/2005) más que tratar de sacar conclusiones erróneamente a partir de reflexiones que van de lo que es, en contraste con lo que debería ser (Moore, 1903/2005).

El utilitarismo y/o consecuencialismo -este último término, por cierto, acuñado por Anscombe (1958) - no requiere una amplia discusión teórica; simplemente define, como criterio deseable, aquel que conduzca a un resultado que maximice las ganancias 
y minimice las pérdidas (Mill, 1863/2005). El utilitarismo es racionalista y considera que los resultados, independientemente de los medios o las intenciones, deben ser la prioridad. Más es mejor que menos.

Hacia la primera mitad del siglo XX, en Cambridge, la postura moral, o amoral, que defendían representantes como Bertrand Russell $(1928,1929)$ y Alfred Ayer $(1936 / 2001)$ era el emotivismo de corte humeano que es una teoría escéptica, incluso nihilista, que sostiene que no existe fundamento ni convicciones morales, excepto lo que el individuo experimente emocionalmente frente a un hecho o una acción. Esta misma postura será retomada en la actualidad por la psicología experimental del estadounidense Joshua Greene et al. (2001, 2004, 2008), entre otros (Haidt, 2001; Prinz, 2004), para tratar de explicar la respuesta de las personas ante los dilemas morales, sin un compromiso, más que los sentimientos inmediatos.

En contraste con lo anterior, el deontologismo define como deseable, no tanto el resultado, como el cumplimiento de un deber moral (Kant, 1788 / 2006). María José Urteaga (2006) afirma en forma preclara:

\begin{abstract}
Muchas son las razones por las que podemos preguntarnos qué es lo que realmente debemos hacer, pero es evidente que esta pregunta cobra más fuerza cuando nuestras identidades prácticas entran en conflicto. Cuando esto sucede, es decir, cuando nuestras obligaciones se contraponen y reflexionamos sobre ello, a lo que apunta nuestra reflexión es a resolver este conflicto a partir de jerarquizar nuestras obligaciones, dando prioridad a las obligaciones que son morales, es decir, dando prioridad a lo que realmente deberíamos hacer (Urteaga, 2006: 278).
\end{abstract}

De esta manera sucinta, aparecen cuatro posturas filosóficas morales: utilitarismo, emotivismo, deontologismo y ética de la virtud. Esto nos servirá para explicar, parcialmente, algunas respuestas frente a los dilemas.

\title{
LOS DILEMAS
}

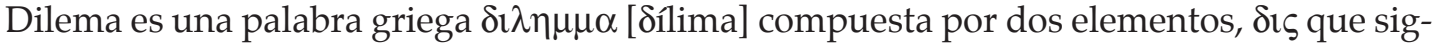
nifica dos y $\lambda \eta \mu \mu \alpha$ que es proposición o premisa (Findlater, 2017). Se presenta un dilema cuando existe un contexto en la cual no se sabe qué se debe hacer o no se tiene una certeza de las consecuencias de una decisión, ya que puede elegirse entre dos opciones -o tres(Di Nucci, 2013) y todas ellas pueden incluir resultados indeseados o no previstos (Platts, 2001).

Los estudios sobre teoría de las decisiones, y en lo general de la comprensión de los mecanismos para la cognición social y la moral (Bechara et al., 2000, 2005; Churchland, 2011; Ciaramelli et al., 2007; Damasio, 1996, 2007; Gazzaniga, 2012; Greene et al., 2002, 2004; Haidt, 2001; Hauser, 2006; Koenigs et al., 2007; Mikhail, 2007; Unger, 1996), muestran un especial interés en el análisis de algunos dilemas morales -ahora clásicos-, dentro de la literatura especializada. Si bien el planteamiento de dilemas y paradojas ha existido desde la Grecia clásica como, por ejemplo, el Dilema de Evatlo (Gelio, clásico/2007), en la didáctica del desarrollo de habilidades de pensamiento complejo (Gómez y Rodríguez, 2018; Heredia, 2013), los dilemas hipotéticos se despliegan con una nueva perspectiva construida desde la imaginación, en donde se pregunta a los participantes de experimentos mentales: «¿Usted qué haría en este escenario?» en forma directa, o de manera indirecta: «¿Usted qué piensa que debería hacerse en esta situación?». Esto se formula así con el propósito, entre otros, de observar, en la práctica, cuáles son los componentes y mecanismos a través de los cuales se construye el juicio moral de las personas. 
La aplicación de test con dilemas morales tiene ya una amplia tradición dentro de los estudios empíricos de las éticas aplicadas. Los estudios para determinar las convicciones morales de las personas van desde saber si estarían a favor o en contra de actuar en situaciones dilemáticas, hasta determinar cuál sería el algoritmo de decisión más apropiado para un automóvil de conducción autónoma, frente a un objeto en su camino (Ballinas-Hernández et al., 2020).

Quisimos saber si es posible llevar estas dudas axiológicas al campo de lo empírico y de lo experimental, y tratar de entender cuáles eran los criterios específicos de los estudiantes a la hora de tomar sus propias decisiones morales. Realizamos un estudio con un instrumento simple, pero que pusiera a prueba sus convicciones. Los resultados se interpretaron bajo los supuestos del utilitarismo, el emotivismo, el deontologismo y la ética de la virtud.

\section{EL ESTUDIO}

Participantes. En el trabajo de campo, los datos del estudio se recopilaron a partir de la aplicación de un instrumento que a continuación se detalla, en dos sedes del Complejo Regional Nororiental de la Benemérita Universidad Autónoma de Puebla.

La primera muestra se obtuvo de alumnos de la Preparatoria «Tlatlauquitepec» (TLA1), quienes cursaban la asignatura de «Filosofía» del Plan 06 de enseñanza media; la segunda muestra fue obtenida de alumnos de la carrera de Nutrición Clínica en el Complejo Universitario de la Salud (CUS1) en Teziutlán, Puebla.

Participaron en total 272 alumnos, menos dos que no respondieron a la totalidad del test, por lo que se anuló su participación y se computaron $n=270$ participantes. La edad promedio de los estudiantes fue de 16 años de edad, de los cuales, 39\% fueron hombres y $61 \%$ fueron mujeres. El 65\% tuvieron 15 años de edad el día que respondieron el test, el 18\% tenía 16 años y, el restante $17 \%$, entre 18 a 20 años.

Instrumento. El instrumento empleado fue un test simple en hoja de tamaño carta, impresa por ambas caras en un solo color, con el título «Test de tres dilemas morales». En la parte superior derecha anotaron la fecha de aplicación; su nombre completo como un dato opcional (o podían dejarlo en blanco); y obligatoriamente, el nivel y grado de estudios. Por último, indicaron su género y edad, escribiendo la edad con número y encerrando en un círculo la letra « $\mathrm{M} »$ para el género masculino $\mathrm{y}$ «F» para el género femenino.

Los dilemas. Utilizamos el Dilema del tranvía (Foot 1967) en adelante Trolley; el Dilema de la pasarela (Thomson, 1985) en adelante Fat, y el Dilema de Sofía (Styron 1979) en adelante Sofía. Tres dilemas clásicos de la psicología experimental, para determinar la justificación y las preferencias de juicio moral.

- Primer dilema: Trolley. «Un tranvía corre fuera de control por una vía. En su camino se hallan cinco personas atadas a la vía por un filósofo malvado. Afortunadamente, es posible accionar un botón que encaminará al tranvía por una vía diferente, por desgracia, hay otra persona atada a esta. ¿Debería pulsarse el botón?». 


\section{Imagen 1. Versión libre inspirada en Foot, 1967}

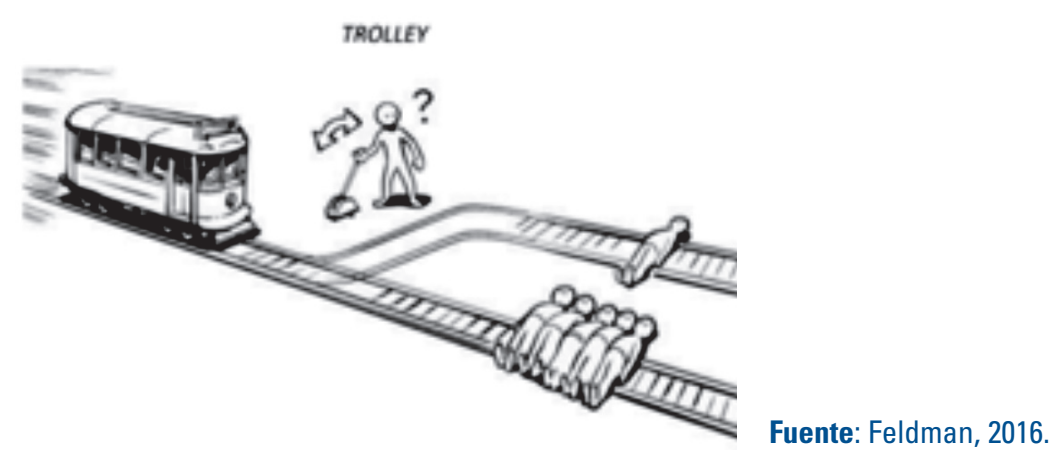

- Segundo dilema: Fat. «Como antes, un tranvía descontrolado se dirige hacia cinco personas. El sujeto se sitúa en un puente sobre la vía y podría detener el paso del tren, lanzando un gran peso delante del mismo. Mientras esto sucede, al lado del sujeto solo se halla un hombre muy gordo; de este modo, la única manera de parar el tren es empujar al hombre gordo desde el puente hacia la vía, acabando con su vida para salvar otras cinco. ¿Qué debe hacer el sujeto?».

\section{Imagen 2.}

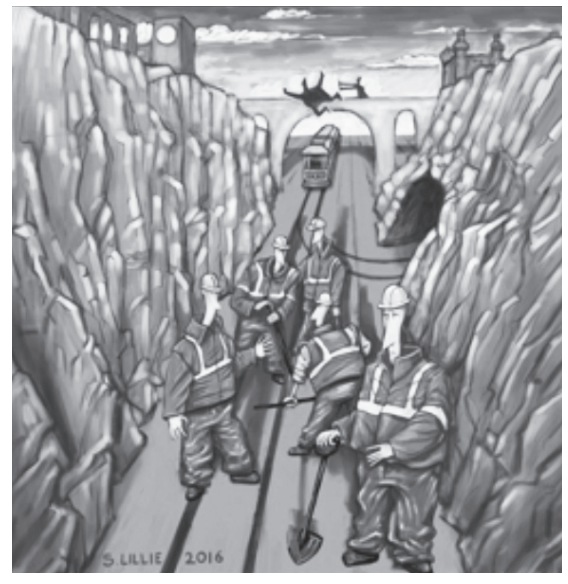

Fuente: Lillie, 2016

- Tercer dilema, Sofía. El tercer dilema se basa en la novela del escritor estadounidense William Styron, llevada al cine por el director Alan J. Pakula, en 1982: «Una madre judía llega a un campo de concentración con sus dos hijos pequeños. Un oficial le pide que elija uno, el cual podría conservar; en caso de que se negara a elegir, matará a ambos. ¿Debe elegir a uno?» (versión libre inspirada en Styron, 1979).

Procedimiento. Inmediatamente después de ser expuestos los dilemas, sigue una pregunta cerrada: «SÍ » o «NO». Posteriormente se formula una pregunta abierta en la que deben responder «¿POR QUÉ?». Esto es: los participantes deben argumentar las razones o el motivo por el que responden de manera afirmativa o negativa; para ello, tienen el espacio en la hoja y el tiempo suficiente para su exposición. No se les delimitó un lapso de tiempo para responder, pero el promedio empleado fue de 15 minutos.

En las tres historias, el participante tiene la posibilidad de contestar con un «SÍ»o con un «NO», lo cual genera ocho posibles combinaciones de respuestas cerradas y, podría 
afirmarse, una gradación del consecuencialismo al deontologismo, así como respuestas conforme al modelo ético virtuoso o el emotivismo.

Existen ocho combinaciones posibles de respuestas:

(1) [SÍ-SÍ-SÍ] significa que: / Sí debe apretar el botón. / Sí debe empujar al hombre. / Sí debe escoger Sofía a uno de sus hijos.

(2) [SÍ-SÍ-NO]: Sí debe apretar el botón. / Sí debe empujar al hombre. / No debe escoger Sofía a uno de sus hijos.

(3) [SÍ-NO-NO]: Sí debe apretar el botón. / No debe empujar al hombre. / No debe escoger Sofía a uno de sus hijos.

(4) [SÍ-NO-Sí]: Sí debe apretar el botón. / No debe empujar al hombre. / Sí debe escoger Sofía a uno de sus hijos.

(5) [NO-NO-NO]: No debe apretar el botón. / No debe empujar al hombre. / No debe Sofía escoger a uno de sus hijos.

(6) [NO-SÍ-NO]: No debe apretar el botón. / Sí debe empujar al hombre. / No debe Sofía escoger a uno de sus hijos.

(7) [NO-NO-SÍ]: No debe apretar el botón. / No debe empujar al hombre. / Sí debe Sofía escoger a uno de sus hijos.

(8) [NO-SÍ-Sí]: No debe apretar el botón. / Sí debe empujar al hombre. / Sí debe Sofía escoger a uno de sus hijos.

A los participantes se les explicó que «no existen respuestas o combinaciones correctas o incorrectas», pero que sí era importante construir algún tipo de justificación a sus respuestas.

\section{PRINCIPALES RESULTADOS}

\section{Resultados cuantitativos}

Los resultados de preferencia de las posibles combinaciones se expresan en la siguiente tabla: el porcentaje de respuestas se observa en la vertical y las posibles combinaciones de respuesta en la horizontal.

Tabla 1. Tres dilemas morales

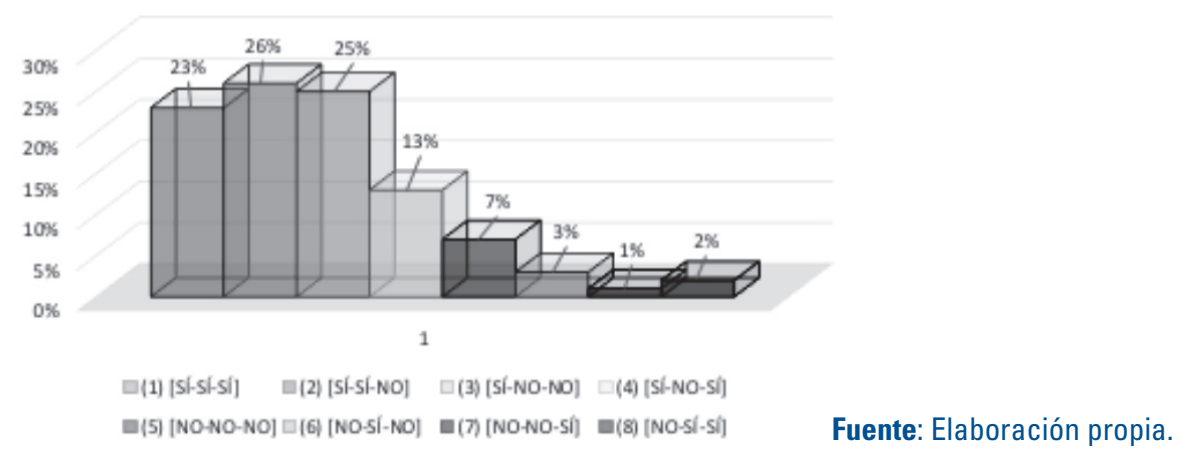


Tabla 2.

\begin{tabular}{|l|l|}
\hline (1) [Sí-Sí-Sí] & $23 \%$ \\
\hline (2) [Sí-Sí-NO] & $26 \%$ \\
\hline (3) [Sí-NO-NO] & $25 \%$ \\
\hline (4) [Sí-NO-Sí] & $13 \%$ \\
\hline (5) [NO-NO-NO] & $7 \%$ \\
\hline (6) [NO-Sí-NO] & $3 \%$ \\
\hline (7) [NO-NO-Sí] & $1 \%$ \\
\hline (8) [NO-Sí-Sí] & $2 \%$ \\
\hline
\end{tabular}

Como observamos, el estudio arrojó resultados mixtos. El 23\% de los participantes respondieron con un (1) [SÍ-SÍ-SÍ] esto es, SÍ debe apretarse el botón para salvar a las cinco personas, a costa de la vida de uno; SÍ debería empujarse al hombre obeso para salvar a los cinco, y Sí debería Sofía escoger a uno de sus hijos. De ese 23\%, no existe una variación significativa $(+-2 \%)$ entre hombres y mujeres. Lo mismo en relación con la edad: no aparece una variación relevante.

Solo un 7\% de los encuestados consideró en los tres dilemas que la respuesta debía ser negativa (5) [NO-NO-NO]; esto es, que NO debería cambiarse el curso del tranvía, que NO debería de empujarse a la persona, y que Sofía NO debería escoger a alguno de sus hijos. En este caso tampoco la variable del género o la edad fueron relevantes, pues de ese $7 \%$, tanto hombres como mujeres, consideraron lo mismo. Tampoco apareció una variación significativa de acuerdo con la edad.

La combinación con mayor número de adherencias $-26 \%$ del total-, fue la combinación (2) SÍ-SÍ-NO esto es, que tanto en Trolley como en Fat, SÍ se debería actuar para salvar a los cinco. Pero cuando se les preguntó sobre qué debe hacer Sofía, consideran que nada, que NO debe escoger a alguno.

Inmediatamente después, la respuesta con mayor adhesión fue la combinación (3) SÍNO-NO así que 25\% de los encuestados creen que Sí debe accionarse el botón para salvar a cinco sacrificando a uno, pero NO debe empujarse al hombre, NI Sofía debe escoger a alguno de sus hijos.

Llama nuestra atención que el porcentaje de la combinación (2) fue ligeramente mayor (26\% sobre $25 \%$ ) de quienes sostuvieron la creencia de que es mejor salvar a cinco personas que a una en ambos casos (3), pero dado el margen de error de $+-2 \%$ que tiene el resultado, podría indicarse que la proporción es casi igual entre quienes cambiaron de parecer entre Trolley y Fat, y quienes no lo hicieron.

Las combinaciones restantes tuvieron bajo porcentaje de adherencias, por ejemplo: (6) [NO-SÍ-NO] con un 3\% que consideró que NO debía cambiarse el curso del tranvía con el botón, pero que Sí debía empujarse a la persona en el puente, pero que Sofía NO debía escoger. Solo un $2 \%$ consideró posible que (8) [NO-SÍ-SÍ] NO debía apretarse el botón, pero que SÍ debía empujarse a la persona y que Sofía Sí debería escoger. La respuesta con menor adherencia 
fue (7) [NO-NO-Sí], únicamente un $1 \%$ consideró -extrañamente- que NO debía pulsar el botón, NO debía empujar a la persona, pero que Sofía Sí debía escoger a alguno de sus hijos.

Algunos resultados como (4) [SÍ-NO-SÍ] representan el 13\% y opinan que Sí debe apretarse el botón, NO se debe empujar al hombre, pero Sofía Sí debe escoger a uno de sus hijos. Lo mismo ocurre con quienes (3\%) escogieron (6) [NO-SÍ-NO] que NO se debía mover la palanca, que Sí debía empujarse al hombre y que Sofía NO debería escoger a ninguno de sus hijos.

\section{Resultados cualitativos}

Como ya se señaló, dividimos el instrumento en dos partes. En la primera parte de la repuesta a los tres dilemas, únicamente los participantes debían responder afirmativamente o negativamente a las preguntas; sin embargo, en la segunda parte de cada razonamiento, deberían responder a la pregunta: «¿POR QUÉ?», con el propósito de que argumentaran sus propias creencias.

La justificación de quienes respondieron mayoritariamente (86\%) SÍ al primer dilema Trolley -esto es, que Sí debía apretarse el botón para cambiar el curso del tranvía para salvar a cinco personas, muriendo en el acto una- fue que: «Es mejor que muera una persona y que se salven cinco vidas».

Al segundo dilema, Fat la justificación se repitió constantemente, en el sentido de la utilidad de un mayor bien, en este caso, igualmente: «Cinco vidas son mejor que solo una», fue de $82 \%$. Y en el caso de Sofía, nuevamente el argumento utilitarista prevaleció (82\%) entre quienes prefirieron la respuesta afirmativa, dado que consideraron, independientemente de otras posibles secuelas no previstas, que era: «Mejor perder a un hijo, que perder a dos».

En los casos de quienes respondieron (7\%) que NO debía apretarse el botón, NO debía empujarse al hombre y Sofía NO debía escoger, la mayoría (73\%) argumentó de forma deontológica que: «No se debe matar porque es malo». En el caso de Sofía la respuesta recurrente en quienes argumentaron que NO era adecuado que lo hiciera fue porque consideraron que: «Sofía después se arrepentiría de haberlo hecho», tal como Styron (1979) lo había predicho en su historia.

Veamos otras respuestas a la pregunta «¿POR QUÉ?». En Trolley, la respuesta de SÍ debe desviarse el tranvía:

«-Porque es preferible que muera una persona a que mueran cinco».

«-El daño sería menor al morir uno que al morir cinco».

«-Mejor cargar en la conciencia a una persona que a cinco».

«-Porque salvaría la vida de cinco y ¡sería un héroe!».

En Trolley, la respuesta de NO debe desviarse el tranvía:

«-Porque es malo matar a una persona inocente».

«-Porque estaría muy nerviosa y no podría hacer nada». 
«-Existe sobrepoblación mundial, así que mueran cinco, no es una gran cosa».

«-Porque el tranvía sigue su curso y el sujeto estaría involucrado en la muerte de alguien y se sentirá culpable, porque se podría decir que decidió la muerte de alguien».

-«¿Quién soy yo para decidir la vida de otros?».

En Fat, la respuesta de SÍ debe empujarse al hombre:

«-Porque de ese modo salvaría la vida de cinco personas».

«-Debería empujarlo porque ya vivió y comió mucho. Así que aquí podría salvar la vida de otros y, como su muerte sería instantánea, no sufriría y serviría de algo».

«-Si él no se cuida, ¿por qué yo habría de hacerlo?».

«-De igual modo, pronto va a morir por su sobrepeso».

En Fat, la respuesta de NO debe empujarse al hombre:

«-Porque el sujeto estaría decidiendo sobre la vida de otra persona, en este caso del hombre gordo. En cambio, si no lo empuja, no sentirá culpa por haber jugado con la vida de otra persona».

«-Porque me daría asco verlo cómo se destroza».

«-Todos tenemos derecho a vivir».

«-Porque estaría cometiendo un asesinato».

En Sofía, la respuesta de SÍ debería escoger a uno de sus hijos:

«-Porque conservar la vida de uno, puede ayudar a mitigar la pérdida del otro».

«-Creo que una madre siempre quiere a sus hijos, pero siempre habrá uno que quiera más. Así que debe escoger y, además, ya no estará [el otro] para reclamarle que por qué no lo escogió».

«-Sería un sacrificio, pero al menos conservaría la vida de uno».

“—¿Para qué dejar que mueran ambos, si puede salvar a uno?".

En Sofía, al responder que NO debería escoger a uno de sus hijos:

«-Porque todos van a morir en el campo de concentración y la madre tiene la opción de suicidarse después de que se los lleve el soldado».

«-Viviría con ese gran remordimiento de conciencia de haber escogido a uno y al otro, no; y se lamentará por eso y estará en sufrimiento. Debe ser el trato igual para sus dos hijos».

«-No escoger, porque así ya no sufrirían en el campo de concentración. Igual van a morir todos». 
«-Porque sabría que escogió a uno y no la dejaría el remordimiento jamás».

«-Si escoge a uno tal vez salve al otro, pero eso sería temporal, porque también morirá y entonces se sentirá mucho más culpable de haber escogido a uno de ellos».

«-El no escoger les evitará a ambos que sean martirizados en el campo de concentración».

\section{DISCUSIÓN}

En las respuestas obtenidas vimos reflejadas las cuatro posturas morales enunciadas. De acuerdo con otros estudios realizados (Di Nucci, 2013; Gold, Pulford y Colman, 2015; Tassy, Oullier, Mancini y Wicker, 2013), los resultados más frecuentes de los tres dilemas han sido, para el caso de Trolley, que la mayoría de los participantes estarían dispuestos a sacrificar a la persona de la vía alterna para salvar a los cinco de la vía principal (Hauser, 2006) esto es, podría interpretarse como una postura utilitarista que, en nuestro caso, se reflejó en las respuestas «-El daño sería menor al morir uno que al morir cinco»; «-Mejor cargar en la conciencia a una persona que a cinco», y «-Porque salvaría la vida de cinco y ¡sería un héroe!».

En cambio, para el caso de Fat resulta que, incluso quienes estaban de acuerdo en sacrificar a uno para salvar a cinco, no estuvieron tan seguros de empujar a la persona para salvar a los cinco, argumentando razones emotivistas: «-Porque me daría asco verlo cómo se destroza». Aunque el resultado fuera el mismo. La mayoría de las personas, según Greene et al. (2004, 2008), no aprueban empujar al hombre para salvar la vida de los cinco.

Finalmente, Sofía lo consideramos un dilema difícil de interpretar, dado que no hay una tendencia histórica clara de su resultado pero, aun no conociendo la novela en que está inspirado, la mayoría de los participantes (82\%) resuelven que Sofía no debería escoger a ninguno de los dos. Sin embargo, en la novela de Styron (1979) y, en la versión llevada a la pantalla (Pakula, 1982), Sofía decide sacrificar a uno de ellos, a la niña, y se queda con el niño de lo cual se arrepentirá toda su vida. Ambas respuestas fueron reflejadas: «-¿Para qué dejar que mueran ambos si puede salvar a uno?»; en contraste con: «-Si escoge a uno tal vez salve al otro, pero eso sería temporal porque también morirá y, entonces, se sentirá mucho más culpable de haber escogido a uno de ellos».

Ahora, volviendo a Greene et al. (2001, 2004, 2008), afirman que la mayoría de las personas están de acuerdo con desviar el tranvía en Trolley como respuesta a un sentido utilitarista de maximización de los resultados. Sin embargo, en el dilema Fat, no ocurre lo mismo. Aunque el resultado podría ser el mismo - salvar la vida de cinco, sacrificando a uno- como se trata de tener contacto físico con la persona, el porcentaje de respuestas afirmativas a la acción de empujarlo disminuye considerablemente, incluso entre quienes habían afirmado recién que utilizarían el dispositivo para desviar el tranvía y salvar a cinco personas, pero - reiteran Greene et al.- no se atreverían a empujar a la persona con el mismo fin, sobre todo porque intervienen posturas emotivistas, por ejemplo: «-Porque estaría muy nerviosa y no podría hacer nada».

Greene et al. (2001, 2008) afirman que existe un sentido emocional más fuerte que una racionalización de principios y, como ya indicamos, aunque el resultado es el mismo, la explicación es distinta. Se actúa en respuesta a las emociones que se disparan. Así, Trolley sería un dilema impersonal, porque no hay contacto físico con la persona y se emplea 
un dispositivo, ya sea un botón o una palanca para desviar indirectamente al tranvía; en cambio, Fat es un dilema personal porque implica contacto físico y directo.

Greene y su equipo fueron más lejos en su laboratorio y llegaron a la conclusión de que las distintas respuestas al daño personal e impersonal tendrían su origen en la actividad de zonas del cerebro específicas diferentes (2008). Ello a partir de la evidencia empírica durante experimentos controlados y con la ayuda de escáner de imágenes cerebrales por resonancia magnética. La conclusión, para Greene et al., es que en el caso de Fat, se activan las áreas cerebrales relacionadas con la cognición social y las emociones, como la corteza prefrontal ventromedial (vmPFC), la amígdala (AMG) y la corteza cingulada posterior. En cambio, en el caso de Trolley, se activan las áreas involucradas en la cognición superior racional, esto es, la corteza prefrontal dorsolateral (DLPFC) y el lóbulo parietal inferior, resultados que también han, y habían sido, estudiados por otros (Fumagalli y Priori, 2012; Martínez-Selva et al., 2006; Albert, Arangüena y Martín, 2010).

La explicación coincidente (Di Nucci, 2013; Gold, Pulford y Colman, 2015; Greene et al., 2001, 2004, 2008; Tassy, Oullier, Mancini y Wicker, 2013) es que en Trolley opera la maximización del resultado con un sentido utilitarista; en cambio en Fat, existe una respuesta emocional que impide asesinar intencionalmente a un inocente, como es el caso del hombre obeso recargado en la barandilla del puente. Greene et al. (2008) argumentan que se disparan ciertas emociones básicas que la biología evolutiva (Churchland, 2011) ha interpretado como un indicio natural que impide que una persona asesine a otra, sin motivo alguno. Para Churchland (2011) existe una evolución biológica que atiende a nuestros antepasados y a la necesidad de no matarnos unos a otros, sino vivir en sociedad, restringiendo la violencia y creando escenarios cooperativos colectivos.

Sin embargo, nos parece que las respuestas son más complejas que un dualismo utilitarismo-emotivismo al que llegan Greene y otros (Di Nucci, 2013; Gold, Pulford y Colman, 2015; Tassy, Oullier, Mancini y Wicker, 2013). Consideramos que, por el contrario, no podemos generalizar o categorizar a los participantes como utilitaristas y emotivistas, porque los resultados nos permitieron visualizar tanto una ética de la virtud como un sentido del cumplimiento de la ley, para determinar la viabilidad argumentativa de ciertas respuestas.

Esto ya había sido visualizado desde el medioevo. Para Tomás de Aquino (I-II, 90-97), la ley pertenece a la razón y, tal como lo señaló Anscombe (1958), para que surja una ley debe existir un legislador, ¿es esto una falacia? Tomás de Aquino argumentó que el canon de la ley entiende que existe una ley natural también llamada ley eterna en la que, de alguna manera, se inspira el derecho positivo y que, con un poco de inteligencia puede comprenderse de forma racional e incluso intuitiva, que no debe lastimarse a nadie sin importar los motivos o los resultados (Barbosa y Jiménez, 2017). De Aquino llamó sindéresis (In sent, 2-24) a la potencia natural con hábito de optar siempre por el bien y evitar el mal. Anscombe lo tradujo, como hemos dicho, a la virtud moral.

La ética de la virtud posee un sentido general, pero a la vez, y aunque podría sonar contradictorio, más específico para evaluar la actuación de las personas. Es actuar por una convicción justificada y, a la vez, por el cumplimiento de un deber moral traducido en la calidad humana. Si bien no hace falta una justificación ulterior del concepto -por ejemplo, de valor intrínseco del derecho a la vida-, puede ser una postura convincente, en contraste con una utilitarista o emotivista contextualizada. No significa tampoco que el utilitarismo milleano no considere aspectos generales de la calidad de las personas, pero se basa en un baremo que es de corte cuantitativo. 


\section{HALLAZGOS}

Después de realizado el análisis general de los datos, los resultados logran demostrar, de manera indirecta, que los jóvenes son capaces de construir juicios morales y justificarlos: el 86\% de los alumnos considera que Sí debería desviarse el tranvía y 82\% que SÍ debe empujarse al hombre. También el $82 \%$ considera que Sofía Sí debe escoger. Lo anterior, hipotéticamente, nos daría un promedio de $83.3 \%$ de potenciales utilitaristas, pero esta generalización sería prematura dado que la combinación de resultados, y sobre todo de las respuestas a las preguntas del ¿POR QUÉ?, arrojan otras interpretaciones menos reduccionistas.

Hubo quien simplemente consideró que matar es malo, Vgr. «-Porque estaría cometiendo un asesinato» $\mathrm{y}$ «-Todos tenemos derecho a vivir», tal como Michel Sandel (2011) argumenta en su obra y curso Justicia...; entonces, bajo estas premisas, las respuestas correctas serían: que NO debe moverse la palanca; NO debe empujarse al hombre, y Sofía NO debe escoger a ninguno. Tomar la vida de otro, así fuera para salvar a cinco o al número que sea, no se justifica desde un punto de vista ético de los derechos; sobre todo porque no es justo para quien es sacrificado (Sandel, 2011).

Lo anterior sería coincidente con una ética de la virtud porque nos habla de la calidad de la persona que no se atrevería a ponerse en el lugar de un legislador (Anscombe, 1958) para decidir quién vive y quién muere: "-Porque el sujeto estaría decidiendo sobre la vida de otra persona, en este caso del hombre gordo. En cambio, si no lo empuja, no sentirá culpa por haber jugado con la vida de otra persona» $\mathrm{y}$ «-Todos tenemos derecho a vivir».

Con un sentido del pathos que representa el acto mismo de matar a otra persona, que bien puede ilustrarse en las respuestas: «-Porque [...] estaría involucrado en la muerte de alguien y se sentirá culpable porque se podría decir que "decidió la muerte de alguien"» o «-Porque sabría que escogió a uno y no la dejaría el remordimiento jamás». La culpa y el remordimiento hablan del sentido de lo moral en estos casos.

Debemos matizar: esto tampoco significa que sostengamos que todos tengan una conciencia moral desarrollada porque, como descubrió Bauman et al. (2014), los dilemas ficticios llegan incluso a provocar ironía y hasta burla entre los participantes jóvenes, especialmente en dilemas como Fat; véanse las respuestas: «-Si él no se cuida, ¿por qué yo habría de hacerlo?» o «-Debería empujarlo, porque ya vivió y comió mucho...» en donde, evidentemente, no se refleja empatía alguna.

Lo que sí hallamos es que es válida la categorización de los cuatro enfoques morales: utilitarismo, deontologismo, emotivismo y ética de la virtud, y que no somos utilitaristas del todo, ni emotivistas permanentes, tampoco deontologistas de corte rigorista o virtuosos per se. No existe un parámetro único, pero ver reflejadas estas posturas, de suyo, posee un valor intrínseco para analizar los distintos caminos para la toma de decisiones con implicaciones morales.

\section{CONCLUSIÓN}

En este documento proporcionamos evidencia empírica a partir de tres dilemas morales clásicos para evaluar el grado en que los jóvenes estudiantes de bachillerato y universitarios 
aprueban el sacrifico de una persona para salvar la vida de cinco, como es el caso de Trolley y Fat; y la conveniencia de escoger a uno de los hijos o perder a ambos, en el caso de Sofía.

Los resultados por supuesto no son concluyentes, aunque arrojan interesantes respuestas por parte de los encuestados, especialmente cuando se les pide justificar sus convicciones, ya sea que se trate de creencias justificadas o creencias inmediatas, sin mayor análisis; el estudio nos proporciona elementos para futuros trabajos con otros dilemas, por ejemplo, de bioética tratándose del Centro Universitario de la Salud (BUAP).

Observamos que las personas no siempre cambian de opinión cuando se interviene en un escenario de manera indirecta, pulsando un botón o empujando una palanca, que cuando lo hacen de manera directa, empujando a una persona a la vía. Los resultados aquí obtenidos muestran que el porcentaje se mantuvo estable en ambos escenarios hipotéticos.

El estudio, repetimos, arrojó resultados mixtos, algunos coincidentes con los metadatos que otros trabajos previos reportaban; sin embargo, constatamos que aunque cuantitativamente en el estudio parezca que los jóvenes son, digamos utilitaristas radicales, no necesariamente se puede generalizar, toda vez que las justificaciones específicas proporcionaron una variación considerable y disímil, entre unos y otros.

Finalmente, los autores nos decantamos en suponer que, tanto la ética de la virtud como el actuar por una convicción y cumplimiento de un deber moral, como sugiere Urteaga, puede ser una postura más convincente moralmente que una postura utilitarista o emotivista contextualizada.

\section{Agradecimientos}

Los autores agradecemos las observaciones realizadas por los revisores externos en el proceso anónimo par ciego, llevadas a cabo en este trabajo, las cuales sirvieron para eliminar erratas y enriquecer el texto. Así mismo a los editores de la Revista Panamericana de Pedagogía por su acompañamiento en el proceso de solventación de las observaciones. También queremos agradecer al Complejo Regional Nororiental de la Benemérita Universidad Autónoma de Puebla, por las facilidades otorgadas para el desarrollo del Proyecto ID: 00687, denominado: Estudio comparativo de las teorías sobre los correlatos biológicos en la toma de decisiones morales, presentado a la Vicerrectoría de Investigación y Estudios de Posgrado (VIEP), en su Convocatoria 2019. 


\section{REFERENCIAS}

Anscombe, G. (1958). Modern moral philosophy. Philosophy, 33(124), 1-19.

Ayer, A.J. (1936/2001). Language, Truth and Logic. NY: Penguin.

Ballinas-Hernández, A., Olmos-Pineda, I., Olvera-López, A. (2020). Detección de irregularidades en las superficies viales para la conducción autónoma en países en vías de desarrollo. Elementos, 118, 23-28. Disponible en: https:/ / elementos.buap.mx/directus / storage/uploads/00000005092.pdf

Barbosa, S. \& Jiménez-Leal, W. (2017). It's not right but it's permitted: Wording effects in moral judgement. Judgment and Decision Making, 12(3), 308. Disponible en: https:// www.sas.upenn.edu/ baron/journal/16/161212c/jdm161212c.pdf

Bauman, C. W., McGraw, A. P., Bartels, D. M. \& Warren, C. (2014). Revisiting external validity: Concerns about trolley problems and other sacrificial dilemmas in moral psychology. Social and Personality Psychology Compass, 8(9), 536-554. https://onlinelibrary. wiley.com/doi/abs/10.1111/spc3.12131

Bechara, A., Tranel, D., Damasio, H. (2000). Characterization of the decision-making impairment of patients with bilateral lesions of the ventromedial prefrontal cortex. $\mathrm{Bra}$ in, 123, 2189-2202. Disponible en: https:/ / www.ncbi.nlm.nih.gov/ pubmed/11050020

Churchland, P. (2011). El cerebro moral. Lo que la neurociencia nos cuenta sobre la moralidad. Barcelona: Paidós.

Ciaramelli, E., Muccioli, M., Làdavas, E. \& di Pellegrino, G. (2007). Selective deficit in personal moral judgment following damage to ventromedial prefrontal cortex. Social cognitive and affective neuroscience, 2(2), 84-92. Disponible en: https://academic.oup. $\mathrm{com} / \mathrm{scan} / \mathrm{article} / 2 / 2 / 84 / 2736768$

Copi, I. M. (1976). Introducción a la lógica. BBAA: Eudeba.

Coutlee, C. G. \& Huettel, S. A. (2012). The functional neuroanatomy of decision making: prefrontal control of thought and action. Brain research, 1428, 3-12. Disponible en: https:/ / www.ncbi.nlm.nih.gov/pmc/articles/PMC3202063/

Damasio, A. (2007). En busca de Spinoza. Neurobiología de la emoción y los sentimientos. Barcelona: Crítica.

Damasio, A. R. (1996). The somatic marker hypothesis and the possible functions of the prefrontal cortex. Philosophical Transactions of the Royal Society of London. Series B: Biological Sciences, 351(1346), 1413-1420. Disponible en: https:/ / royalsocietypublishing. org/doi/abs/10.1098/rstb.1996.0125

Di Nucci, E. (2013). Self-sacrifice and the trolley problem. Philosophical Psychology, 26(5), 662-672. Disponible en: https://www.tandfonline.com/doi/abs/10.1080/09515089.20 $\underline{12.674664}$

Edmonds, D. (2013). Would you kill the fat man? The trolley problem and what your answer tells us about right and wrong. Princeton: Princeton University Press.

Feldman, B. (2016). The Trolley Problem Is the Internet's Most Philosophical Meme [imagen web]. https://nymag.com/intelligencer/2016/08/trolley-problem-meme-tumblr-philosophy.html 
Findlater, A. (2017). Chambers's etymological dictionary of the English language. Chambers.

Foot, P. (1967). The problem of abortion and the doctrine of double effect. Oxford Review 5, 5-15. DOI: 10.1093/0199252866.003.0002. Disponible en: http:/ / www.oxfordscholarship.com/view/10.1093/0199252866.001.0001/acprof-9780199252862-chapter-2

Fumagalli, M. \& Priori, A. (2012). Functional and clinical neuroanatomy of morality. Brain, 135(7), 2006-2021. Disponible en: https://academic.oup.com/brain/article $/ 135 / 7 / 2006 / 350263$

Gazzaniga, M. S. (2012). ¿Quién manda aquí? El libre albedrío y la ciencia del cerebro. Barcelona: Paidós.

Gelio, A. (2007). Noches áticas. Madrid: Alianza Editorial.

Gold, N., Pulford, B. D. \& Colman, A. M. (2014). The outlandish, the realistic, and the real: contextual manipulation and agent role effects in trolley problems. Front Psychol, (5) 35, 1-10. Disponible en: http:/ / dx.doi.org/10.3389/fpsyg.2014.00035

Gómez, M.; Rodríguez, R. (2018). Estrategia didáctica para la comprensión de dilemas morales con apoyo en las TIC. Infancias Imágenes, 18(1), 9-20. Disponible en: https:// dialnet.unirioja.es/descarga/articulo/6992026.pdf

Greene, J. \& Haidt, J. (2002). How (and Where) does Moral Judgment Work? Cognitive Science, 6, 517-523. Disponible en: http:/ / people.stern.nyu.edu/jhaidt/articles/greene. haidt.2002.moral-judgment.pub024.pdf

Greene, J. D. (2002). The terrible, horrible, no good, very bad truth about morality and what to do about it [Tesis doctoral inédita]. Princeton: Princeton University.

Greene, J. D., Morelli, S. A., Lowenberg, K., Nystrom, L. E. \& Cohen, J. D. (2008). Cognitive load selectively interferes with utilitarian moral judgment. Cognition, 107(3), 1144-1154. Disponible en: https://www.sciencedirect.com/science/article/abs/pii/ $\underline{\mathrm{S} 0010027707002752}$

Greene, J., Nystrom, L., Engell, A., Darley, J. \& Cohen, J. (2004). The Neural Bases of Cognitive Conflict and Control in Moral Judgment. Neuron, 44, 389-400. Disponible en https:// www.sciencedirect.com/science/article/pii/S0896627304006348

Greene, J., Sommerville, R.B., Nystrom, L., Darley, J.M. \& Cohen, J.D. (2001). An fMRI investigation of emotional engagement in moral judgment. Science, 293(5537), 21052108. DOI: 10.1126/science.1062872. Disponible en: https://www.ncbi.nlm.nih.gov/ pubmed/11557895

Greene, Joshua D. (2009). Dual-process morality and the personal/impersonal distinction: A reply to McGuire, Langdon, Coltheart, and Mackenzie. Journal of Experimental Social Psychology 45(3), 581-584. Disponible en: https://dash.harvard.edu/hand$\underline{\mathrm{le} / 1 / 4264762}$

Haidt, J. (2001). The emotional dog and its rational tail: a social intuitionist approach to moral judgment. Psychological review, 108(4), 814. Disponible en: http:/ / citeseerx.ist. psu.edu/viewdoc/download?doi=10.1.1.124.9206\&rep=rep1\&type $=$ pdf

Hart, H.L.A. (1967). Intention and Punishment. En Punishment and Responsibility: Essays in the Philosophy of Law, 113-135. Oxford: Oxford University Press. Disponible en: https:// doi.org/10.1093/acprof:oso/9780199534777.003.0005 
Hauser, M. (2006). Moral minds: How nature designed our universal sense of right and wrong. Harper Collins Publishers.

Heredia, Y. (2013). Los dilemas morales como estrategia de enseñanza- aprendizaje para el desarrollo moral en los alumnos de preescolar. Revista de Investigación Educativa del Tecnológico de Monterrey, 4(7), 61-69. Disponible en: https://core.ac.uk/download/ pdf/71081505.pdf

Lillie, S. (2016). Illustrations and various artworks [Imagen]. Steve Lillie. Fuente: http:// www.stevelillie.biz/editorial/8vc03zlrohxhfwn5twhrp6njpmp13a

Kant, I. (1788/2006). Crítica de la razón práctica. Madrid: Sígueme.

Koenigs, M., Young, L., Adolphs, R., Tranel, D., Cushman, F., Hauser, M. \& Damasio, A. (2007). Damage to the prefrontal cortex increases utilitarian moral judgements. Nature, 446(7138), 908-911. Dsiponible en: http:/ / www.pef.uni-lj.si/kognitivna/DOCUMENTS/Izbirni izpit/koenigs et al.pdf

Martínez-Selva, J. M., Sánchez-Navarro, J. P., Bechara, A. y Román, F. (2006). Mecanismos cerebrales de la toma de decisiones. Revista de neurología, 42(7), 411-418. Disponible en: https:/ / dialnet.unirioja.es/ servlet/articulo?codigo=1961794

Mikhail, J. (2007). Universal moral grammar: Theory, evidence and the future. Trends in cognitive sciences, 11(4), 143-152. Disponible en: https:// www.sciencedirect.com/science/article/abs/pii/S1364661307000496

Mill, J. S. (1863/2005). On Liberty. Cambridge Texts in the History of Political Thought. Cambridge: Cambridge University Press.

Moore, G. E. (1903/2005). Principia Ethica. NY: Barnes \& Noble Books.

Morandín-Ahuerma, F. (2019). ¿Quién mató a Elaine? Autos robot y toma de decisiones. Elementos, 115, 33-38. Disponible en: https://elementos.buap.mx/directus/storage/ uploads/00000003966.pdf

Pakula, A. (1982). Sophie's choice [Film]. Los Angeles: Universal Pictures.

Platts, M. (Comp.) (2001). Dilemas éticos. México: UNAM.

Prinz, J. J. (2004). Gut reactions: A perceptual theory of emotion. New York: Oxford University Press.

Russell, B. (1928/2011). Sceptical Essays. London: George Allen \& Unwin.

Russell, B. (1929/2001). Marriage and Morals. London: George Allen \& Unwin.

Salerno, g. (2007). Rigorismo y pluralidad de principios en ética. Anuario Filosófico, 40, 697-718. Disponible en: https:// revistas.unav.edu/index.php/anuario-filosofico/article/view/29252/0

Sandel, M. J. (2011). Justicia: ¿Hacemos lo que debemos? Madrid: Debate.

Styron, W. (1979). Sophie's Choice. New York: Random House.

Tassy, S., Oullier, O., Mancini, J. \& Wicker, B. (2013). Discrepancies between judgment and choice of action in moral dilemmas. Front Psychol, (4)250, 1-8. http://dx.doi. org/10.3389/fpsyg.2013.00250 
Thomson, J. (1976). Killing, letting die, and the trolley problem. The Monist, 59(2), 204217. https:// doi.org/10.5840/monist197659224.

Thomson, J. (1985). The Trolley Problem. The Yale Law Journal, 94(6), 1395-1415. DOI: 10.2307/796133. Disponible en: https://digitalcommons.law.yale.edu/cgi/viewcontent.cgi?referer=https: / / www.google.com / \&httpsredir=1\&article=6930\&context=ylj

Unger, P. K. (1996). Living high and letting die: Our illusion of innocence. Oxford: Oxford University Press.

Urteaga Rodríguez, M. J. (2016). Desobediencia civil: la autoridad de la reflexión vs. la autoridad civil. International Journal of Philosophy, (3), 274-284. Disponible en: https:// dialnet.unirioja.es/descarga/articulo/5523227.pdf

Weber, M. (1919/2011). La política como vocación. NoBooks Editorial.

Welzel, H. (1951). Zum Notstandsproblem. Zeitschrift für die gesamte Strafrechtswissenschaft 63, 47-56. doi: 10.1515/zstw.1951.63.1.47. Disponible en: https:// www.degruyter. com/view/j/zstw.1951.63.issue-1/zstw.1951.63.1.47/zstw.1951.63.1.47.xml

Wittgenstein, L. (1921/2012). Tractatus Logico Philosophicus. Simon and Schuster. 